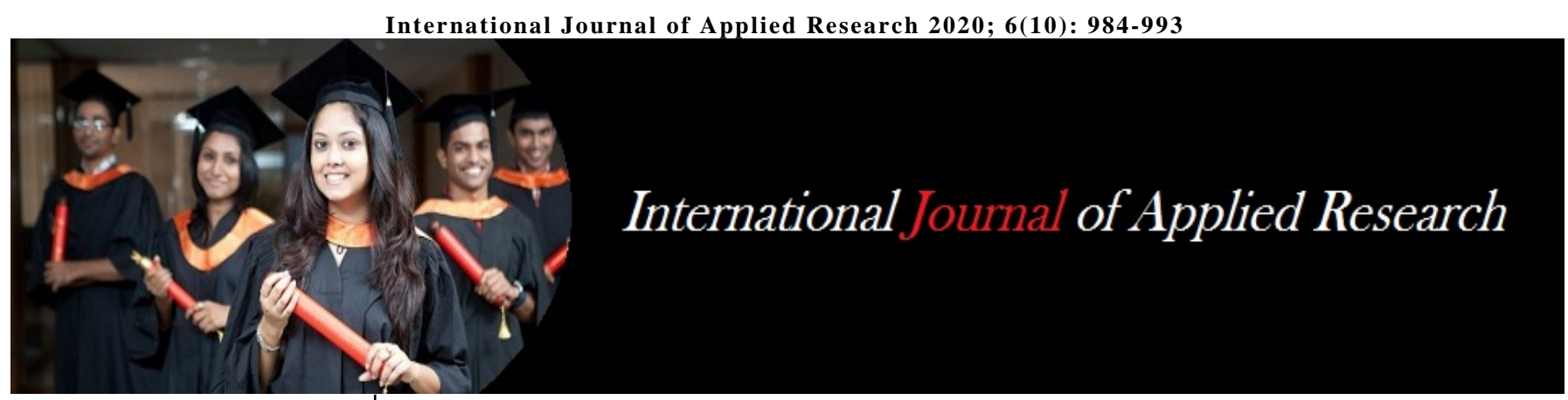

ISSN Print: 2394-7500 ISSN Online: 2394-5869 Impact Factor: 8.4 IJAR 2020; 6(10): 984-993 www.allresearchjournal.com

Received: 18-06-2018 Accepted: 22-07-2018

Dr. Naima Umar Ph.D, Post-Doctoral Fellow Department of Geography, AMU, Aligarh, Uttar Pradesh, India
Corresponding Author: Dr. Naima Umar Ph.D, Post-Doctoral Fellow Department of Geography, AMU, Aligarh, Uttar Pradesh, India

\section{Sources of irrigation in the state of Uttar Pradesh: A regional analysis}

\section{Dr. Naima Umar}

DOI: https://doi.org/10.22271/allresearch.2020.v6.i10o.7681

\section{Abstract}

Agriculture is the primary occupation in India which supports by irrigation facilities. Irrigation sources are not even everywhere in the state of Uttar Pradesh. It has variations in terms of plenty of water supply and many parts of the state are facing scarcity. Assured irrigation plays important role in increasing the yield of any area. After the introduction of new agricultural technology within Uttar Pradesh has resulted in the advancement in the use of NPK fertilizers, insecticides, weedicides which has raised the demand for water supply for growing crops. However, overuse of groundwater has led to a significant drop in the water table in the state. The present paper has discussed the sources of irrigation such as canal irrigation, tubewell irrigation, etc. in the state of Uttar Pradesh by taking average values during the period from 1996-2000 and 2001-2005 and also highlighted the regional variations in the district of Uttar Pradesh.

Keywords: Agriculture, Technology, Irrigation, Water Supply, Ground Water.

\section{Introduction}

Water is an important input for successful agriculture. Water may be available to crops by rainfall, and it can also supply to fields through human efforts. The process of supplying water to crops by means of canals, well, tube-wells, tanks, ponds or tapping water from underground sources. Irrigation has been one of the most important factors which in increasing agricultural production, as the propagation green revolution technology was entirely dependent upon it. It is realized that assured and controlled water supply is an essential requirement for achieving the full potentials of high-yielding varieties (HYV) of seeds. It is estimated that, more than five tonnes of water is needed to grow one kilogram of rice. B.D. Dhawan (1980) has illustrated that the vast Gangetic basin of Easten Uttar Pradesh is well known for its abundence of water resources, both surface water and ground water. New agricultural technology has increased groundwater irrigation many fold, with advancement in well-established canal irrigation system (Sivanappan, 1995).

\section{Objectives}

1. To categorize the state in into very high, high, medium, low and very low categories.

2. To highlight the areas to be focuss on.

3. To draw attention and suggest suitable measures for the purpose to improve weaker districts of the state.

\section{Material and Methods}

Present paper has discussed about the changing irrigation pattern during 1996 to 2000 and 2001 to 2005. It has been carefully tabulated for 10 year huge data, is divided into two periods of time from 1996-2000 and from 2001-2005 by taking average values. Final values have been calculated on the basis of percentage method. Present paper is based on secondary sources of data.Data were obtained from the Directorate of Agricultural Statistics and Crop Insurance (Krishi Bhawan), Lucknow, U.P. 


\section{Results and Discusion}

\section{(a) Net irrigated area}

Intensity of irrigation can be considered in terms of percentage share of net irrigated area to the net cropped area. The extent of net irrigated area shows an increase of 77.81 per cent during the period of 2001-05 as compared to 1996-2000 which was 77.19 per cent in the state. The highest intensity of irrigation is seen in the district of Hathras which has 97.35 per cent irrigated area and the lowest intensity of irrigation found in the district of Sonbhadra which has 24.81 per cent net irrigated area. The districts of the state were categorized for irrigation intensity into five categories of very high, high, medium, and low and very low intensity of irrigation area.

Table. 1 shows that during 1996-2000 there were 15 districts of Muzaffarnagar, Baghpat, Gaziabad, Bulandshahar, Aligarh, Mathura, Hathras, Etah, Firozabad, Mainpuri, Kannauj Rampur, Pilibhit, Mau and Chandauli having very high net irrigated area above 87.44 per cent. There were 19 districts of Saharanpur, Meerut, J. P. Nagar, G. B. Nagar, Bareilli, Budaun, Shahjahanpur, Farrukhabad, Agra, Barabanki, Lucknow, Kannauj, Raebareli, Azamgarh Ghazipur, Jaunpur, Pratapgarh, Kaushambi and S. R. Nagar characterized with high net irrigated area with a values in between 76.07 and 87.44 per cent.

Medium intensity of net irrigated area found in 15 districts of Bijnor, Moradabad, Hardoi, Kanpur (urban), Kanpur (rural), Lalitpur, Maharajganj, Kushi Nagar, Basti, S. K. Nagar, Gorakhpur, Deoria, Ballia, Sultanpur and Varanasi which have ranged in between 61.56 and 76.07 per cent.

Table 1: Net arrigated area in Uttar Pradesh - 1996-2000

\begin{tabular}{|c|c|c|c|}
\hline Category & Index range & No. of districts & Name of the district \\
\hline Very High & Above 87.44 & 15 & $\begin{array}{r}\text { Muzaffarnagar Baghpat, Gaziabad, Bulandshahar, Aligarh, Mathura, Hathras, Etah, Firozabad, } \\
\text { Mainpuri, Kannauj Rampur, Pilibhit, Mau and Chandauli }\end{array}$ \\
\hline High & $76.07-87.44$ & 19 & $\begin{array}{c}\text { Saharanpur, Meerut, J. P. Nagar, G. B. Nagar, Bareilli, Budaun, Shahjahanpur, Farrukhabad, Agra, } \\
\text { Barabanki, Lucknow, Kannauj, Raebareli, Azamgarh Ghazipur, Jaunpur, Pratapgarh, Kaushambi } \\
\text { and S. R. Nagar }\end{array}$ \\
\hline Medium & $61.56-76.07$ & 15 & $\begin{array}{r}\text { Bijnor, Moradabad, Hardoi, Kanpur (urban), Kanpur (rural), Lalitpur, Maharajganj, Kushi Nagar, } \\
\text { Basti, S. K. Nagar, Gorakhpur, Deoria, Ballia, Sultanpur and Varanasi }\end{array}$ \\
\hline Low & $31.4-61.56$ & 15 & $\begin{array}{r}\text { Kheri, Sitapur, Shrawasti, Balrampur, Siddarthnagar, Goada, Faizabad, Etawah, Jalaun, Jhansi, } \\
\text { Mahoba, Fatehpur, Banda, Allahabad and Mirzapur }\end{array}$ \\
\hline Very Low & Below 31.4 & 6 & Bahraich Ambedkar Nagar, Auraiya, Hamirpur, Chitrakut and Sonbhadra \\
\hline
\end{tabular}

Low intensity of net irrigated area shown by 15 districts of Kheri, Sitapur, Shrawasti, Balrampur, Siddarthnagar, Goada, Faizabad, Etawah, Jalaun, Jhansi, Mahoba, Fatehpur, Banda, Allahabad and Mirzapur with ranged in between 31.4 and 61.56 per cent and very low net irrigated area found in the 6 districts of Bahraich Ambedkar Nagar, Auraiya, Hamirpur, Chitrakut and Sonbhadra have net irrigated area below 31.4 per cent (Fig. 1).

During 2001-2005, Fig. 2 shows that the area under net irrigation has increased. There are 16 districts of
Saharanpur, Muzaffarnagar, Baghpat, Meerut, Ghaziabad, J. P. Nagar, Rampur, Pilibhit, Aligarh Mathura, Hathras, Etah, Firozabad, Mainpuri, Ambedkar Nagar and Chandauli, characterized with very high area under net irrigation above 91.66 per cent.

High net irrigated area spread in 14 districts of Bulandshahar, Budaun, Bareilly, Shahjahanpur, Haradoi, Kannauj, Barabanki, Lucknow, Unnao, Mau, Azamgarh, Jaunpur, Pratapgarh and Varanasi with a value in between 84.66 and 91.66 per cent.

Table 2: Net Irrigated Area in Uttar Pradesh - 2001-2005

\begin{tabular}{|c|c|c|c|}
\hline Category & Index range & No. of districts & Name of the district \\
\hline Very High & Above 91.66 & 16 & $\begin{array}{c}\text { Saharanpur, Muzaffarnagar, Baghpat, Meerut, Ghaziabad, J. P. Nagar, Rampur, } \\
\text { Pilibhit, Aligarh Mathura, Hathras, Etah, Firozabad, Mainpuri, Ambedkar Nagar and } \\
\text { Chandauli, }\end{array}$ \\
\hline High & $84.66-91.66$ & 14 & $\begin{array}{c}\text { Bulandshahar, Budaun, Bareilly, Shahjahanpur, Hardoi, Kannauj, Barabanki, } \\
\text { Lucknow, Unnao, Mau, Azamgarh, Jaunpur, Pratapgarh and Varanasi }\end{array}$ \\
\hline Medium & $71.67-84.66$ & 22 & $\begin{array}{c}\text { Bijnor, Moradabad, Kheri, Sitapur, Farrukhabad, Agra, Etawah, Auraiya, Kanpur } \\
\text { (rural), Lalitput, Faizabad, Sultanput, Raebareli, Maharajganj Kushi Nagar, S. K. } \\
\text { Nagar, Gorakhpur, Deoria, Mau, Ghazipur, S. R. Nagar and Allahabad. }\end{array}$ \\
\hline Low & $48.94-71.67$ & 11 & $\begin{array}{c}\text { Bahraich, Siddharth Nagar, Gonda, Basti, G. B. Nagar, Jalaun, Jhansi Kanpur (urban), } \\
\text { Fatehpur, Khushambi and Mirzapur }\end{array}$ \\
\hline Very Low & Below 48.94 & 7 & Shrawasti, Balrampur, Hamirpur, Mahoba, Banda, Chitrakut and Sonbhadra \\
\hline
\end{tabular}

There were 22 districts of Bijnor, Moradabad, Kheri, Sitapur, Farrukhabad, Agra, Etawah, Auraiya, Kanpur (rural), Lalitput, Faizabad, Sultanput, Raebareli, Maharajganj, Kushi Nagar, S. K. Nagar, Gorakhpur, Deoria, Mau, Ghazipur, S. R. Nagar and Allahabad characterized with medium area under net irrigation with a value ranged in between 71.67 and 84.66 per cent. Low net irrigated area with a value in between 48.94 and 71.67 per cent demarcated 11 districts namely Bahraich, Siddharth Nagar, Gonda, Basti, G. B. Nagar, Jalaun, Jhansi Kanpur (urban), Fatehpur, Khushambi and Mirzapur and very low net irrigated area found in 7 districts of Shrawasti, Balrampur, Hamirpur, Mahoba, Banda, Chitrakut and Sonbhadra with a value below 48.94 per cent. 


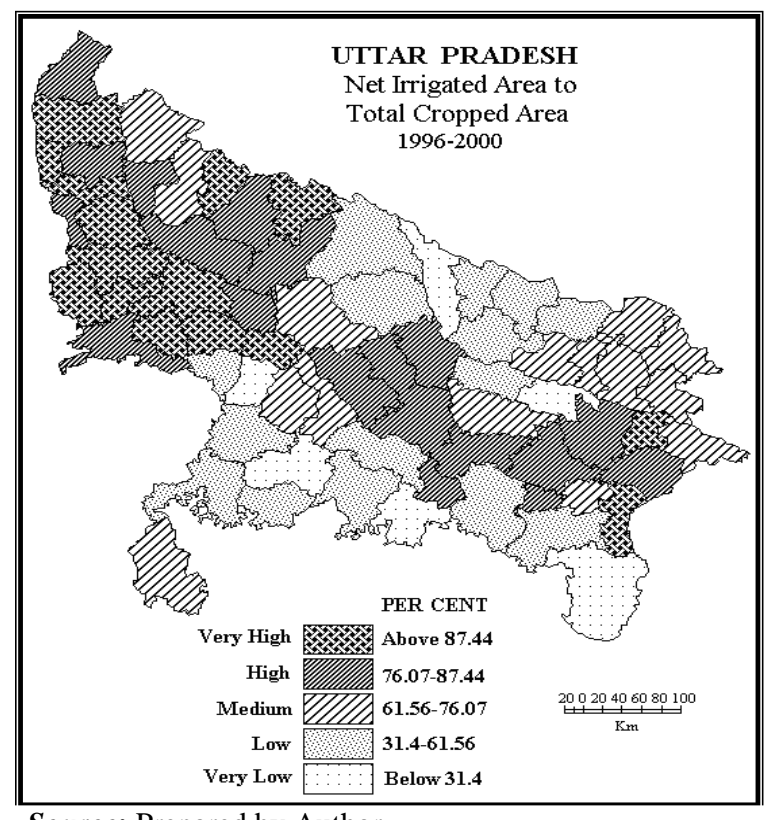

Source: Prepared by Author

Fig 1: Uttar Pradesh Net irrigated area to total cropped area 19962000

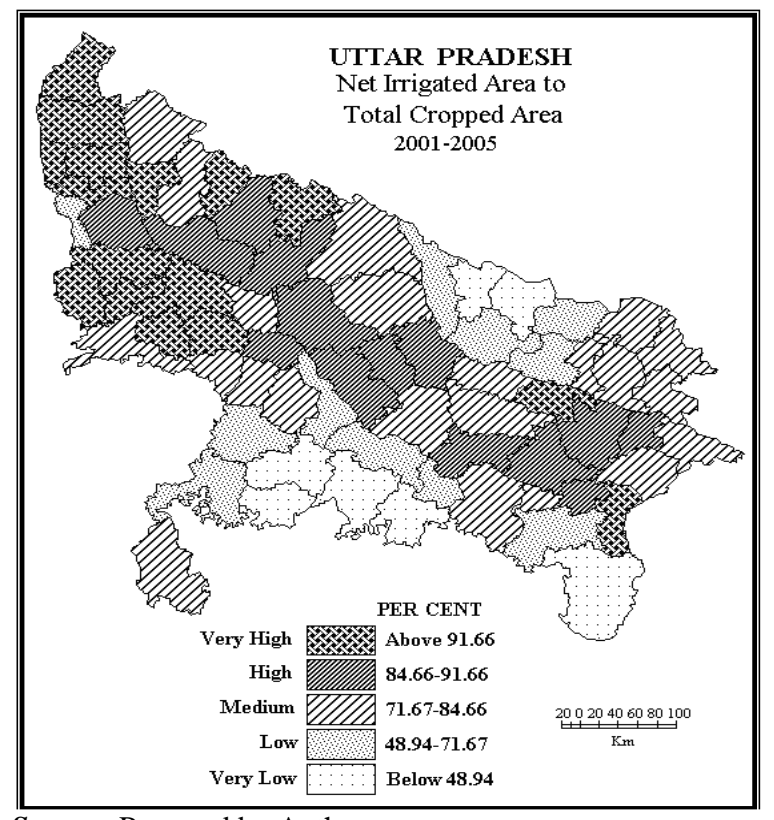

Source: Prepared by Author

Fig 2: Uttar Pradesh Net irrigated area to total cropped area 20012005

\section{(b) Area under canal irrigation}

Canals constitute an important source of irrigation in the state of Uttar Pradesh. The state is drained by perennial rivers originating in the snow covered Himalayan ranges and is blessed with fertile soils. Following are the main canals.
i) Upper Ganga Canal
ii) Lower Ganga Canal
iii) Sharda Canal
iv) Eastern Yamuna Canal
v) Agra Canal
vi) Betwa Canal.

\section{(i) Upper Ganga canal}

This Canal takes off from the Ganga river at Kankhal (Haridwar). The construction of the canal commenced in
1842 and completed in 1854 during the british period. Main canal is $342 \mathrm{~km}$ long, while the length of its distributaries is about 6,200 Km. It irrigates about 7 lakh hectares of land in the upper part of the Ganga-Yamuna doab. Districts of Saharanpur, Meerut, Gaziabad, Bulandshahar, Agra, Mathura, Etah, Kanpur, Mainpuri, Farrukhabad and Fatehpur get benefited from the canal.

Its main branches are namely, Devbandh Anupshahar, Motta and Hathras. It joins with the Lower Ganga canal at Mainpuri from where the quantity of water in canal is considerably increased.

\section{(ii) Lower Ganga canal}

This canal was taken from the river Ganga near Narora (Bulandshahar) in 1878. The length of canal including its distributaries is about $6000 \mathrm{Km}$. Its main branches are known by the names of Etawah, Kanpur, and Fatehpur. It irrigates about 4.6 lakh hectares of agricultural lands in the districts of Bulandshahar, Farrukhbad Mainpuri, Aligarh, Etah, Etawah, Fatehpur, Kanpur and Allahabad.

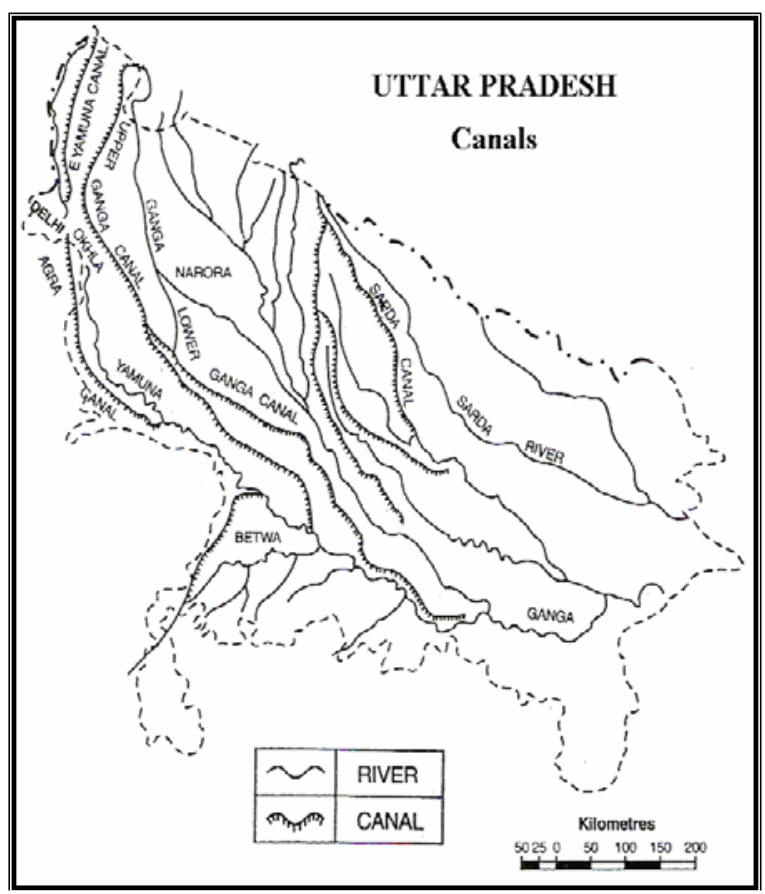

Source: Khullar, D.R. (2006), India: A Comprehensive Geography, p.505

Fig 3: Uttar Pradesh canals

\section{(iii) Sharda canal}

This canal is taken from the Sharda river at Banbasa near the Indo-Nepal border. The construction work on this canal was completed in 1928. The total length of the main canal and its distributaries is $13,624 \mathrm{Km}$. It is thus one of the longest canal systems of the world, and this system irrigates about 8 lakh hectares of land, mainly in the districts of Allahabad, Sultanput, Pilibhit, Bareilly, Hardoi, Shajahanpur, Sitapur, Lucknow, Barabanki, Raebareli, Unnao, Pratapgarh, and Kheri.

\section{(iv) Eastern Yamuna canal}

It takes off from the river Yamuna at Faizabad. It was constructed in 1831. the main canal and its distributaries cover a distance of 1,450 Km and irrigate about 2 lakh hectares of land in the districts of Saharanpur, Mazaffarnagar, Meerut and Ghaziabad. It again joins the 
Yamuna river at Delhi, and irrigates a part of the Union Territory also.

\section{(v) Agra canal}

This canal is taken from the right bank of the Yamuna river at Okhla (Delhi). It was built in 1874 and irrigates about 1.5 lakh hectares in the districts of Agra and Mathura in U.P., Faridabad in Haryana state, Bharatpur in Rajasthan and some parts of Union Territory of Delhi.

\section{(vi) Betwa canal}

Built during the Third Five Year Plan, this canal takes off from the Betwa river about $56 \mathrm{Km}$ southwest of Jhansi. It irrigates about 1.2 lakh hectares in Jhansi, Jalaun and
Hamirpur districts.

Apart from the above major canals, some other canals suchas Ken, Chambal, Dhasan and Son also irrigate some areas in the southern parts of the state of Uttar Pradesh.

Area under canal irrigation in the state has declined from 25.15 per cent during $1996-2000$ to 20.68 per cent during 2001-2005. During 1996-2000, there were only 5 districts of Jalaun, Banda, Chandauli, Mirzapur and Sonbhadra characterized with very high area under canal irrigation above 55.45 per cent. High percentage of area under canal irrigation in between 37.7 and 55.45 per cent is found in 12 districts of Mathura, Etawah, Kanpur (rural), Kanpur (urban), Jhansi, Hamirpur, Mahoba, Barabanki, Raebareli, Pratapgarh, Allahabad, and Varanasi.

Table 3: Canal Irrigated Area in Uttar Pradesh - 1996-2000

\begin{tabular}{|c|c|c|c|}
\hline Category & Index range & $\begin{array}{c}\text { No. of } \\
\text { districts }\end{array}$ & Name of the district \\
\hline Very High & Above 55.45 & 5 & Jalaun, Banda, Chandauli, Mirzapur and Sonbhadra. \\
\hline High & $37.7-55.45$ & 12 & $\begin{array}{c}\text { Mathura, Etawah, Kanpur (rural), Kanpur (urban), Jhansi, Hamirpur, Mahoba, Barabanki, } \\
\text { Raebareli, Pratapgarh, Allahabad, and Varanasi. }\end{array}$ \\
\hline Medium & $22.21-37.7$ & 16 & $\begin{array}{c}\text { Saharanpur, Muzaffarnagar, Pilibhit, Mainpuri, Auraiya, Lalitpur, Lucknow, Unnao, } \\
\text { Fatehpur, Chitrakut, Maharajganj, Khushi Nagar, Sultanpur, Jaunpur, Ballia and Ghazipur. }\end{array}$ \\
\hline Low & $8.9-22.21$ & 20 & $\begin{array}{c}\text { Baghpat, Meerut, Ghaziabad, G. B. Nagar, Bulandshahar, Aligarh, Hathras, Etah, Agra, } \\
\text { Firozabad, Bareilly, Sitapur, Hardoi, Deoria Faizabad, Ambedkar Nagar, Azamgarh, Mau, } \\
\text { Kashambi and S. R. Nagar }\end{array}$ \\
\hline Very Low & Below 8.9 & 17 & $\begin{array}{c}\text { Bijnor, J. P. Nagar, Moradabad, Rampur, Badaun, Farrukhabad, Kannauj, Shahjahanpur, } \\
\text { Kheri, Bahraich, Shrawasti, Balrampur Siddharth Nagar, Gonda, Basti, S. K. Nagar, and } \\
\text { Gorakhpur }\end{array}$ \\
\hline
\end{tabular}

Medium percentage of area under canal irrigation ranged in between 22.21 and 37.7 per cent was marked in 16 districts of Saharanpur, Muzaffarnagar, Pilibhit, Mainpuri, Auraiya, Lalitpur, Lucknow, Unnao, Fatehpur, Chitrakut, Maharajganj, Khushi Nagar, Sultanpur, Jaunpur, Ballia and Ghazipur. There were 20 districts in which low percentage of irrigated area under canals ranged in between 8.9 and 22.21 per cent were namely, Baghpat, Meerut, Ghaziabad, G. B. Nagar, Bulandshahar, Aligarh, Hathras, Etah, Agra, Firozabad, Bareilly, Sitapur, Hardoi, Deoria Faizabad, Ambedkar Nagar, Azamgarh, Mau Kashambi and S. R. Nagar, and 17 other districts characterized with very low percentage of area under canal irrigation below 8.9 per cent were namely, Bijnor, J. P. Nagar, Moradabad, Rampur, Badaun, Farrukhabad, Kannauj, Shahjahanpur, Kheri,
Bahraich, Shrawasti, Balrampur Siddharth Nagar, Gonda, Basti, S. K. Nagar and Gorakhpur.

During the period 2001-2005, irrigated area under canals further declined. Very high percentage of irrigated area above 49.9 per cent under canals was found in the districts of Jalaun, Banda, Allahabad, Mirzapur Chandauli and Sonbhandra, and the districts of Mathura, Etawah, Kannauj, Kanpur (rural), Hamirpur, Jhansi, Kushi Nagar, Barabanki, Raebareli, Pratapgarh, and Chitrakut marked with irrigated area under canals ranged in between 32.3 and 49.9 per cent. Medium irrigated area under canals in between 19.67 and 32.3 per cent was shown by 13 districts of Muzaffarnagar, Pilibhit, Mainpuri, Lucknow, Unnao, Kanpur (urban), Fatehpur, Mahoba, Lalitpur, Sultanpur, Jaunpur, Azamgarh and Ghazipur.

Table 4: Canal Irrigated Area in Uttar Pradesh -2001-2005

\begin{tabular}{|c|c|c|c|}
\hline Category & Index range & No. of districts & Name of the district \\
\hline Very High & Above 49.9 & 6 & Jalaun, Banda, Allahabad, Mirzapur Chandauli and Sonbhandra \\
\hline High & $32.3-49.9$ & 11 & $\begin{array}{c}\text { Mathura, Etawah, Kannauj, Kanpur (rural), Hamirpur, Jhansi, Kushi Nagar, Barabanki, Raebareli, } \\
\text { Pratapgarh, and Chitrakut }\end{array}$ \\
\hline Medium & $19.67-32.3$ & 13 & $\begin{array}{r}\text { Muzaffarnagar, Pilibhit, Mainpuri, Lucknow, Unnao, Kanpur (urban), Fatehpur, Mahoba, } \\
\text { Lalitpur, Sultanpur, Jaunpur, Azamgarh and Ghazipur. }\end{array}$ \\
\hline Low & $7.97-19.67$ & 24 & $\begin{array}{c}\text { Saharanpur, Meerut, Ghaziabad, G. B. Nagar, Bulandshahr, Aligarh, Hathras, Etah, Agra, } \\
\text { Firozabad, Bareilly, Sitapur, Hardoi, Kannauj, Siddharthanagar, Maharajganj, Deoria, Faizabad, } \\
\text { Ambedkar Nagar, Mau, Ballia Varanasi, S. R. Nagar and Kaushambi }\end{array}$ \\
\hline Very Low & Below 7.97 & 16 & $\begin{array}{r}\text { Baghpat, Bijnor, J. P. Nagar, Moradabad, Rampur, Budaun, Shahjahanpur, Farrukhabad, Kheri, } \\
\text { Bahraich, Shrawasti, Balrampur, Gonda Basti, S. K. Nagar and Gorakhpur }\end{array}$ \\
\hline
\end{tabular}

There were 24 districts characterized with low percentage of irrigated area in between 7.97 and 19.67 per cent were namely, sSaharanpur, Meerut, Ghaziabad, G. B. Nagar, Bulandshahr, Aligarh, Hathras, Etah, Agra, Firozabad, Bareilly, Sitapur, Hardoi, Kannauj, Siddharthanagar, Maharajganj, Deoria, Faizabad, Ambedkar Nagar, Mau,
Ballia Varanasi, S. R. Nagar and Kaushambi and the districts of Baghpat, Bijnor, J. P. Nagar, Moradabad, Rampur, Budaun, Shahjahanpur, Farrukhabad, Kheri, Bahraich, Shrawasti, Balrampur, Gonda Basti, S. K. Nagar and Gorakhpur were having very low, irrigated area under canals below 7.97 per cent. 


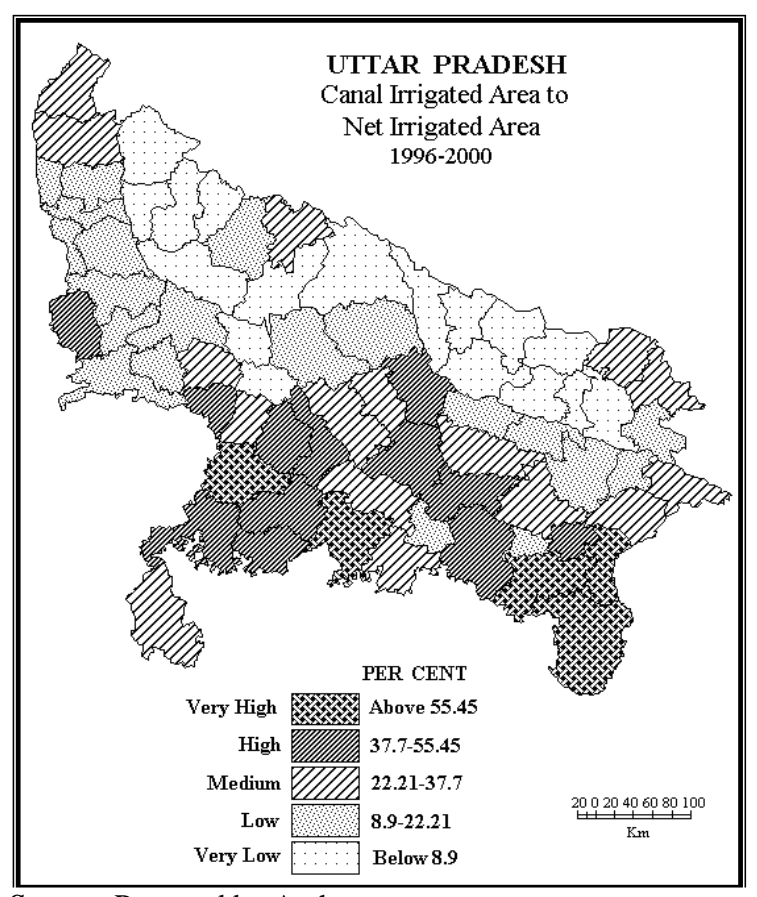

Source: Prepared by Author

Fig 4: Uttar Pradesh canal irrigated area to Net irrigated area 1996-2001

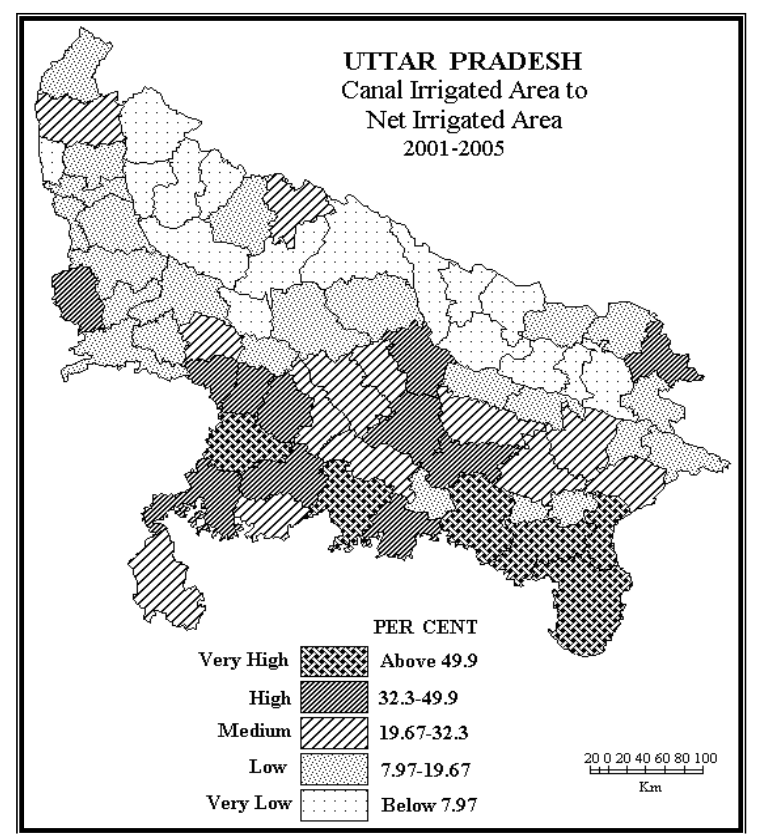

Source: Prepared by Author

Fig 5: Uttar Pradesh canal irrigated area to Net irrigated area 2001-2005

Table 5: Area under Sources of Irrigation in Uttar Pradesh-1996-2000 and 2001-2005

\begin{tabular}{|c|c|c|c|c|c|c|c|c|}
\hline \multirow{2}{*}{ Name of district } & \multicolumn{2}{|c|}{ Net Irrigated Area } & \multicolumn{2}{|c|}{ Irea under Canal Irrigation } & \multicolumn{2}{|c|}{ Irea under Tube-Well Irrigation Area under Other Sources irrigation } \\
\cline { 2 - 10 } & $\mathbf{1 9 9 6 - 2 0 0 0}$ & $\mathbf{2 0 0 1 - 0 5}$ & $\mathbf{1 9 9 6 - 2 0 0 0}$ & $\mathbf{2 0 0 1 - 0 5}$ & $\mathbf{1 9 9 6 - 2 0 0 0}$ & $\mathbf{2 0 0 1 - 0 5}$ & $\mathbf{1 9 9 6 - 2 0 0 0}$ & $\mathbf{2 0 0 1 - 0 5}$ \\
\hline Saharanpur & 86.45 & 91.66 & 27.16 & 18.68 & 72.78 & 81.29 & 0.01 & 0.02 \\
\hline Muzaffarnagar & 94.56 & 97.89 & 29.99 & 24.77 & 69.96 & 75.10 & 0.02 & 0.01 \\
\hline Meerut & 81.33 & 94.51 & 21.49 & 19.45 & 81.97 & 93.81 & 0.01 & 0.02 \\
\hline Bagpat & 96.72 & 96.25 & 10.66 & 6.14 & 78.10 & 80.46 & 0.08 & 0.08 \\
\hline Bulandshahar & 89.26 & 89.16 & 15.22 & 12.21 & 76.19 & 67.68 & 0.26 & 0.05 \\
\hline Ghaziabad & 95.98 & 92.86 & 17.75 & 15.33 & 89.40 & 65.96 & 1.15 & 4.57 \\
\hline G. B. Nagar & 87.41 & 71.57 & 14.23 & 12.86 & 82.43 & 94.59 & 0.13 & 0.07 \\
\hline Aligarh & 96.71 & 98.11 & 14.96 & 11.03 & 90.92 & 91.11 & 1.16 & 0.59 \\
\hline Hatharas & 97.35 & 99.02 & 8.9 & 9.3 & 72.72 & 80.82 & 9.01 & 4.09 \\
\hline Mathura & 93.36 & 98.09 & 41.38 & 38.74 & 73.60 & 68.20 & 0.07 & 0.60 \\
\hline Agra & 79.07 & 84.08 & 16.05 & 10.41 & 91.33 & 92.63 & 0.09 & 0.07 \\
\hline Firozabad & 91.94 & 96.51 & 12.88 & 10.54 & 83.22 & 85.93 & 6.39 & 0.31 \\
\hline Mainpuri & 94.68 & 97.73 & 29.67 & 26.31 & 82.10 & 86.47 & 6.91 & 0.64 \\
\hline
\end{tabular}




\begin{tabular}{|c|c|c|c|c|c|c|c|c|}
\hline Etah & 88.35 & 95.02 & 15.67 & 11.85 & 90.91 & 88.84 & 4.28 \\
\hline Bareilly & 84.23 & 89.94 & 15.27 & 13.34 & 77.99 & 85.05 & 53 \\
\hline Budaun & 85.46 & 90.5 & 0.06 & 0.02 & 69.42 & 75.53 & 70.08 \\
\hline Shahjahanpur & 84.97 & 88.27 & 5.87 & 5.09 & 66.16 & 78.16 & 8.07 \\
\hline Pilibhit & 90.24 & 96.43 & 25.82 & 23.54 & 42.43 & 52.26 & 4.12 & 0.90 \\
\hline Bijnor & 74.97 & 82.87 & 2.2 & 3.67 & 80.08 & 82.28 & 1.23 \\
\hline
\end{tabular}

Table 5: (contd.)

\begin{tabular}{|c|c|c|c|c|c|c|c|c|}
\hline Moradabad & 67.33 & 84.03 & 5.33 & 3.79 & 77.70 & 79.68 & 0.00 & 0.03 \\
\hline J. P. Nagar & 87.08 & 95.04 & 0.05 & 0 & 75.82 & 76.65 & 0.49 & 0.01 \\
\hline Rampur & 87.44 & 93.6 & 4.88 & 1.4 & 7.25 & 12.75 & 0.32 & 1.97 \\
\hline Farrukhabad & 82.1 & 82.28 & 6.15 & 2.74 & 8.72 & 0.30 & 6.85 & 7.43 \\
\hline Kannauj & 89.27 & 90.52 & 6.5 & 10.86 & 19.08 & 21.29 & 3.00 & 8.38 \\
\hline Etawah & 54.56 & 81.98 & 54.94 & 49.6 & 43.43 & 45.71 & 1.34 & 2.21 \\
\hline Auraiya & 0 & 81.44 & 30.69 & 43.76 & 15.63 & 25.57 & 16.99 & 19.48 \\
\hline Kanpur (urban) & 73.73 & 68.54 & 37.7 & 24.01 & 18.45 & 25.84 & 6.11 & 4.79 \\
\hline Kanpur (rural) & 66.44 & 73.29 & 52.92 & 40.44 & 0.81 & 1.41 & 19.66 & 19.95 \\
\hline Fatehpur & 61.08 & 64.06 & 29.97 & 22.73 & 3.84 & 4.15 & 7.99 & 7.93 \\
\hline Allahabad & 61.52 & 71.67 & 54.24 & 49.9 & 13.97 & 17.33 & 1.25 & 2.14 \\
\hline Kaushambi & 82.97 & 68.99 & 10.91 & 16.1 & 48.71 & 55.97 & 0.13 & 0.22 \\
\hline Pratapgarh & 78.11 & 85.76 & 42.91 & 43.25 & 44.15 & 49.98 & 0.19 & 0.20 \\
\hline Jhansi & 48.11 & 61.26 & 48.3 & 42.71 & 81.43 & 88.44 & 0.63 & 0.33 \\
\hline Lalitpur & 66.51 & 80.13 & 33.27 & 32.17 & 58.32 & 61.18 & 0.20 & 0.05 \\
\hline Jalaun & 45.21 & 53.24 & 80.47 & 74.42 & 84.03 & 88.71 & 0.08 & 0.04 \\
\hline Hamirpur & 25.43 & 33.66 & 46.1 & 34.71 & 49.60 & 45.72 & 15.65 & 0.61 \\
\hline Mahoba & 49.76 & 44.11 & 43.66 & 30.26 & 86.89 & 88.04 & 0.27 & 0.09 \\
\hline Banda & 31.4 & 34.28 & 67.87 & 62.43 & 90.18 & 94.43 & 3.51 & 1.08 \\
\hline Chitrakut & 25.09 & 27.59 & 32.94 & 34.55 & 92.67 & 85.97 & 3.19 & 0.67 \\
\hline Varanasi & 72.92 & 84.66 & 46.48 & 11.48 & 84.14 & 97.81 & 10.41 & 1.17 \\
\hline Chandauli & 89.2 & 92.84 & 55.45 & 84.25 & 87.22 & 85.72 & 0.06 & 0.02 \\
\hline Ghazipur & 76.97 & 82.91 & 23.57 & 23.14 & 82.69 & 80.14 & 0.06 & 0.10 \\
\hline Jaunpur & 77.7 & 86.76 & 27.75 & 27.94 & 52.33 & 86.24 & 0.54 & 0.13 \\
\hline Mirzapur & 59.48 & 56.51 & 74.87 & 63.11 & 81.75 & 80.15 & 0.22 & 0.03 \\
\hline Sonbhadra & 24.81 & 24.06 & 82.57 & 85.95 & 69.86 & 71.99 & 2.38 & 0.05 \\
\hline S. R. Nagar & 76.07 & 79.75 & 10.53 & 18.85 & 64.70 & 72.28 & 0.09 & 0.06 \\
\hline Azamgarh & 83.06 & 90.35 & 17.09 & 19.67 & 56.87 & 56.62 & 0.05 & 0.08 \\
\hline
\end{tabular}

Table 5: (contd.)

\begin{tabular}{|c|c|c|c|c|c|c|c|c|}
\hline Mau & $\mathbf{8 8 . 5 2}$ & $\mathbf{8 8 . 1 3}$ & $\mathbf{1 2 . 6 9}$ & $\mathbf{1 1 . 6 4}$ & $\mathbf{8 0 . 5 7}$ & $\mathbf{8 3 . 6 4}$ & $\mathbf{0 . 0 8}$ & $\mathbf{0 . 1 7}$ \\
\hline Ballia & 71.35 & 78.1 & 22.21 & 17.25 & 84.93 & 97.79 & 12.52 & 0.44 \\
\hline Gorakhpur & 74.57 & 80.37 & 5.4 & 3.65 & 84.92 & 84.01 & 0.07 & 0.12 \\
\hline Maharajganj & 71.34 & 79.89 & 29.43 & 18.04 & 55.84 & 67.26 & 0.35 & 0.10 \\
\hline Deoria & 61.56 & 84.42 & 18.09 & 13.29 & 49.33 & 53.75 & 0.28 & 0.06 \\
\hline Kushi Nagar & 66.65 & 74.85 & 31.82 & 36 & 68.97 & 76.80 & 0.67 & 0.43 \\
\hline Basti & 65.15 & 63.19 & 2.94 & 0 & 32.02 & 35.94 & 4.70 & 4.58 \\
\hline Siddharth Nagar & 55.32 & 66.05 & 5.37 & 15.97 & 71.78 & 85.58 & 12.01 & 0.75 \\
\hline S. K. Nagar & 66.51 & 83.05 & 1.6 & 2.3 & 80.01 & 82.32 & 0.53 & 0.22 \\
\hline Lucknow & 82.82 & 89.41 & 27.84 & 20.55 & 71.33 & 78.86 & 0.66 & 0.32 \\
\hline Unnao & 82.83 & 89.53 & 33.92 & 25.69 & 64.25 & 73.29 & 1.70 & 0.44 \\
\hline Raebareli & 80.86 & 84.65 & 50.3 & 46.18 & 60.89 & 74.93 & 0.92 & 0.30 \\
\hline Sitapur & 54.64 & 78.03 & 15.28 & 7.97 & 46.60 & 58.96 & 0.36 & 0.10 \\
\hline Hardoi & 76.02 & 87.93 & 18.44 & 16.35 & 90.71 & 92.88 & 0.49 & 0.08 \\
\hline Kheri & 59.24 & 77.66 & 8.27 & 6.68 & 88.21 & 88.54 & 0.24 & 0.08 \\
\hline Faizabad & 46.98 & 84.34 & 14.99 & 15.8 & 66.68 & 73.65 & 0.17 & 0.01 \\
\hline Ambedkar Nagar & 0 & 94.08 & 12.73 & 14.27 & 87.10 & 89.05 & 0.03 & 0.05 \\
\hline Sultanpur & 72.46 & 80.64 & 35.11 & 27.62 & 85.15 & 90.68 & 0.00 & 0.00 \\
\hline Barabanki & 78.86 & 88.31 & 42.39 & 32.3 & 72.82 & 60.69 & 1.85 & 2.15 \\
\hline Gonda & 51.65 & 67.7 & 0.98 & 0.81 & 91.91 & 88.47 & 8.01 & 3.59 \\
\hline Balrampur & 37.42 & 37.8 & 5.96 & 6.16 & 79.43 & 81.39 & 3.37 & 0.48 \\
\hline Bahraich & 28.05 & 48.94 & 5.58 & 5.06 & 67.03 & 78.05 & 2.52 & 0.01 \\
\hline Shravasti & 55.12 & 39.74 & 0.44 & 1.01 & 1.58 & 10.90 & 27.73 & 22.85 \\
\hline Uttar Pradesh & 70.19 & 77.81 & 25.15 & 20.68 & 67.79 & 71.57 & 3.13 & 1.70 \\
\hline
\end{tabular}

Source: Data obtained from the Directorate of Agriculture, U.P., Lucknow

\section{(c) Area under tube-well irrigation}

Well is a hole dug in the ground to obtain subsoil water. Tube-well irrigation is most important among the sources of irrigation. Intensity of irrigation through tube-well has exceeded from 67.79 per cent during 1996-2000 to 71.57 per cent during 2001-2005 in the state. (Fig. 6.) 
Table 6: Tube-well Irrigated Area in Uttar Pradesh -1996-2000

\begin{tabular}{|c|c|c|c|}
\hline Category & Index range & No. of districts & Name of the district \\
\hline $\begin{array}{l}\text { Very } \\
\text { High }\end{array}$ & Above 85.15 & 13 & $\begin{array}{l}\text { Moradabad, Rampur, Badaun, Hathras, Firozabad, Farrukhabad, Kannauj, Kheri Shrawasti, S. K. } \\
\text { Nagar, Gorakhpur, Ambedkar Nagar and Mau }\end{array}$ \\
\hline High & 73.6 & 21 & $\begin{array}{c}\text { Baghpat, Meerut, Bijnor, J. P. Nagar, Bulandshahar, Aligarh, Agra, Pilibhit, Shahjahanpur, } \\
\text { Hardoi, Bahraich, Balrampur, Gonda, Basti, Deoria, Faizabad, Azamgarh, Ballia, Ghazipur, } \\
\text { Kaushambi and S. R. Nagar }\end{array}$ \\
\hline Medium & 56.87- 73.6 & 17 & $\begin{array}{c}\text { Saharanpur, Muzaffarnagar, Ghaziabad, Mathura, Etah, Mainpuri, Bareilly, Sitaput, Lucknow, } \\
\text { Unnao, Kanpur (urban), Fatehpur, Siddharth Nagar, Maharajganj, Sultanpur, Pratapgarh and } \\
\text { Jaunpur. }\end{array}$ \\
\hline Low & $19.08-56.87$ & 11 & $\begin{array}{l}\text { G. B. Nagar, Etawah, Auraiya, Kanpur (rural), Hamirpur, Barabanki, Raebareli, Kushi Nagar, } \\
\text { Varanasi, Mirzapur and Allahabad }\end{array}$ \\
\hline Tery Low & Below 19.08 & 8 & Jalaun. Jhansi, Lilitput, Mahoba, Banda, Chitrakut, Chandauli and Sonbhadra. \\
\hline
\end{tabular}

Districtwise variations in tube-well irrigation can be seen from Figs. 6.3 and 6.4. During 1996-2000, there were 13 districts namely, Moradabad, Rampur, Badaun, Hathras, Firozabad, Farrukhabad, Kannauj, Kheri Shrawasti, S. K. Nagar, Gorakhpur, Ambedkar Nagar and Mau in them irrigation was provided above 85.15 per cent with the help of tube wells.

There were 21 other districts of Baghpat, Meerut, Bijnor, J. P. Nagar, Bulandshahar, Aligarh, Agra, Pilibhit, Shahjahanpur, Hardoi, Bahraich, Balrampur, Gonda, Basti,
Deoria, Faizabad, Azamgarh, Ballia, Ghazipur, Kaushambi and S. R. Nagar characterized with high irrigated area under tube well ranged in between 73.6 and 85.15 per cent. Medium irrigated area by tube wells in between 56.87 and 73.6 per cent was found in 17 districts of Saharanpur, Muzaffarnagar, Ghaziabad, Mathura, Etah, Mainpuri, Bareilly, Sitapur, Lucknow, Unnao, Kanpur (urban), Fatehpur, Siddharth Nagar, Maharajganj, Sultanpur, Pratapgarh and Jaunpur.

Table 7: Tube-well Irrigated Area in Uttar Pradesh -2001-2005

\begin{tabular}{|c|c|c|c|}
\hline Category & Index range & No. of districts & Name of the district \\
\hline Very High & Above 89.05 & 10 & $\begin{array}{c}\text { Baghpat, J. P. Nagar, Rampur, Hathras, Kheri, Farrukhabad, Firozabad, Gonda, Basti and } \\
\text { Gorakhpur }\end{array}$ \\
\hline High & $78.86-89.05$ & 25 & $\begin{array}{c}\text { Saharanpur, Meerut, Bulandshahar, Aligarh, Agra, Bareilly, Badaun, Shahjahanpur, } \\
\text { Sitapur, Hardoi, Kannauj, Lucknow, Bahraich, Shrawasti, Balrampur, S. K. Nagar, } \\
\text { Deoria, Faizabad, Ambedkar Nagar, Azamgarh, Mau, Ballia, Varanasi, S. R. Nagar and } \\
\text { Kaushambi. }\end{array}$ \\
\hline Medium & $61.18-78.86$ & 16 & $\begin{array}{l}\text { Muzaffarnagar, Bijnor, Moradabad, Ghaziabad, Mathura, Pilibhit, Mainpuri Barabanki, } \\
\text { Unnao, Kanpur (urban), Fatehpur, Siddharthnagar, Maharajganj, Sultanpur, Jaunpur and } \\
\text { Ghazipur. }\end{array}$ \\
\hline Low & $25.84-61.18$ & 11 & $\begin{array}{c}\text { G. B. Nagar, Etah, Etawah, Aurauja, Kanpur (rural), Haminpur, Banda, Kushi Nagar, } \\
\text { Raebareli, Pratapgarh and Allahabad }\end{array}$ \\
\hline Very Low & Below 25.84 & 8 & Jalaun, Jhansi, Lalitpur, Mahoba, Chitrakut, Chandauli, Mirzapur and Sonbhadra. \\
\hline
\end{tabular}

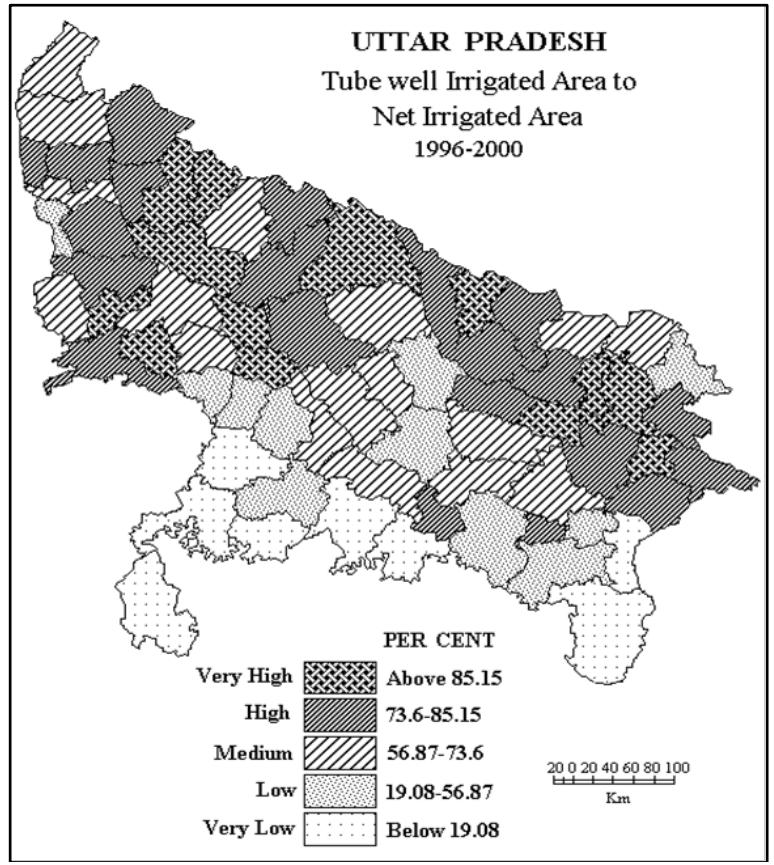

Source: Prepared by Author

Fig 6: Uttar Pradesh Tube well irrigated area to net irrigated area 1996-2000

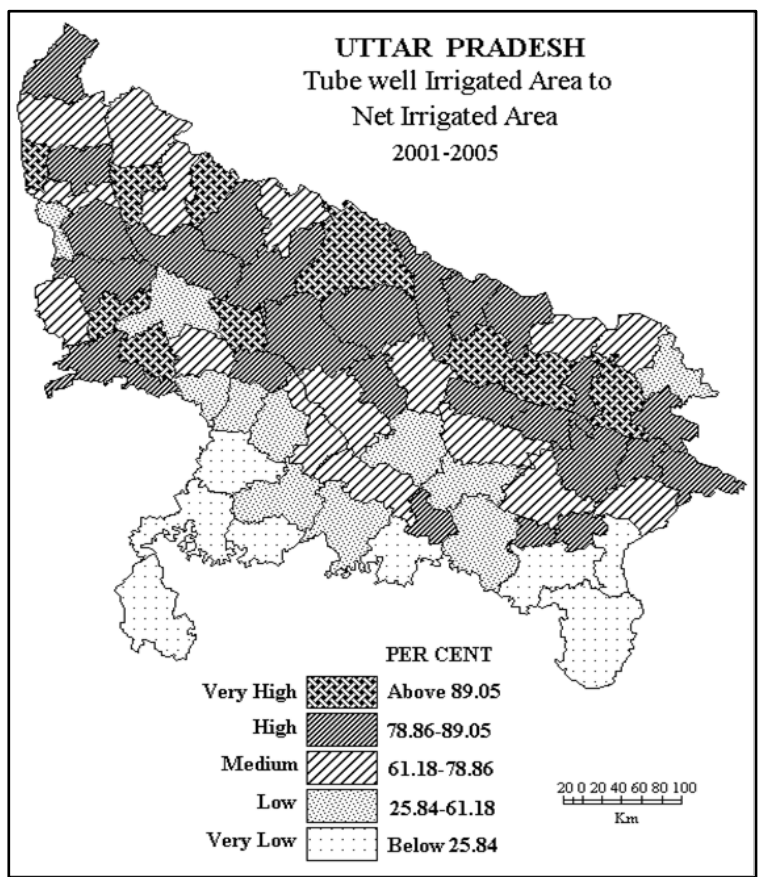

Source: Prepared by Author

Fig 7: Uttar Pradesh Tube well irrigated area to net irrigated area 20001-2005 
As many as 11 districts of G. B. Nagar, Etawah, Auraiya, Kanpur (rural), Hamirpur, Barabanki, Raebareli, Kushi Nagar, Varanasi, Mirzapur and Allahabad marked with low irrigation intensity in tube-well irrigated area, which ranged in between 19.08 and 56.87 per cent. Very low irrigated area under tube-well irrigation below 19.08 per cent was seen in 8 districts of Jalaun. Jhansi, Lilitput, Mahoba, Banda, Chitrakut, Chandauli and Sonbhadra.

Fig. 7 clearly shows that area under tube-well irrigation increased during 2001-2005. Very high percentage of area under tube-well irrigation with a value above 89.05 per cent was in 10 districts of Baghpat, J. P. Nagar, Rampur, Hathras, Kheri, Farrukhabad, Firozabad, Gonda, Basti and Gorakhpur and a high percentage of tube well irrigated areas in between 78.86 and 89.05 per cent was in 25 districts of Saharanpur, Meerut, Bulandshahar, Aligarh, Agra, Bareilly, Badaun, Shahjahanpur, Sitapur, Hardoi, Kannauj, Lucknow, Bahraich, Shrawasti, Balrampur, S. K. Nagar, Deoria, Faizabad, Ambedkar Nagar, Azamgarh, Mau, Ballia, Varanasi, S. R. Nagar and Kaushambi. Medium irrigation intensity by tube-wells in between 61.18 and 78.86 per cent was marked in 16 districts of Muzaffarnagar, Bijnor, Moradabad, Ghaziabad, Mathura, Pilibhit, Mainpuri Barabanki, Unnao, Kanpur (urban), Fatehpur, Siddharthnagar, Maharajganj, Sultanpur, Jaunpur and Ghazipur.

Low percentage of area under tube-well irrigation in between 25.84 and 61.18 per cent was for 11 districts of G. B. Nagar, Etah, Etawah, Aurauja, Kanpur (rural), Haminpur, Banda, Kushi Nagar, Raebareli, Pratapgarh and Allahabad and very low percentage below 25.84 per cent for 8 southern districts of the state namely, Jalaun, Jhansi, Lalitpur, Mahoba, Chitrakut, Chandauli, Mirzapur and Sonbhadra.

\section{(d) Area under other sources of irrigation}

Irrigation by other sources is provided in crop cultivation where canals and tube-wells do not constitute as the source of irrigation. Other sources which provide irrigation are tanks, lakes, ponds, and other wells. Area under other sources of irrigation in the state declined from 3.13 per cent during (1996-2000) to 1.70 per cent during (2001-2005).

During 1996-2000, very high percentage of area irrigated by other sources above 12.52 per cent was marked in 5 districts of G. B. Nagar, Gonda, Lalitpur, Mahoba and Chitrakut.

High percentage of area under other sources of irrigation in between 6.91 and 12.52 per cent was seen in 8 districts of Bareilly, Budaun, Sitapur, Baraich, Balrampur, Siddharthanagar, Basti, and Jhansi. Medium irrigation intensity under other sources in between 4.12 - 6.91 found in 6 districts of Shahjahanpur, Bahraich, Maharajganj, Hamirpur, Banda and Soubhdra and low in 9 district of Ghaziabad, Bulandshahar, Etah, Unnao, S. K. Nagar, Gorakhpur, Jaunpur, Allahabad and Mirzapur ranged in between 1.34 and 4.12 per cent.

Table 8: Other Sources Irrigated Area in Uttar Pradesh -1996-2000

\begin{tabular}{|c|c|c|c|}
\hline Category & Index range & No. of districts & Name of the district \\
\hline Very High & Above 12.52 & 5 & G. B. Nagar, Gonda, Lalitpur, Mahoba and Chitrakut \\
\hline High & $6.91-12.52$ & 8 & Bareilly, Budaun, Sitapur, Baraich, Balrampur, Siddharthanagar, Basti, and Jhansi \\
\hline Medium & $4.12-6.91$ & 6 & Shahjahanpur, Bahraich, Maharajganj, Hamirpur, Banda and Soubhdra \\
\hline Low & $1.34-4.12$ & 9 & Ghaziabad, Bulandshahar, Etah, Unnao, S. K. Nagar, Gorakhpur, Jaunpur, Allahabad and Mirzapur \\
\hline & & & $\begin{array}{c}\text { Saharanpur, Muzaffarnagar, Baghpat, Meerut, Bijnor, J. P. Nagar, Moradabad, Rampur, Pilibhit, } \\
\text { Kheri, Aligarh Mathura, Hathras, Agra, Firozabad, Mainpuri, Farrukhbad, Hardoi, Kannauj, } \\
\text { Auraiya, Etawah, Jalaun, Kanpur (rural), Kanpur (urban), Barabanki, Lucknow, Raebareli, } \\
\text { Fatehpur, Kushi Nagar, Faizabad, Ambedkar Nagar, Sultanpur, Pratapgarh, Kaushambi, Deoria, } \\
\text { Azamgarh, Mau, Ballia, Ghazipur, Sant Ravidas Nagar, Varanasi and Chandauli. }\end{array}$ \\
\hline
\end{tabular}

Very low area below 1.34 per cent under other source irrigation spreads over the 42 districts of Saharanpur, Muzaffarnagar, Baghpat, Meerut, Bijnor, J. P. Nagar, Moradabad, Rampur, Pilibhit, Kheri, Aligarh Mathura, Hathras, Agra, Firozabad, Mainpuri, Farrukhbad, Hardoi, Kannauj, Auraiya, Etawah, Jalaun, Kanpur (rural), Kanpur (urban), Barabanki, Lucknow, Raebareli, Fatehpur, Kushi Nagar, Faizabad, Ambedkar Nagar, Sultanpur, Pratapgarh, Kaushambi, Deoria, Azamgarh, Mau, Ballia, Ghazipur, Sant Ravidas Nagar, Varanasi and Chandauli.
During 2001-2005 as visible from Fig. 9 that only 4 districts having very high area under other sources of irrigation above 8.38 per cent are namely, Mahoba, Lalitpur, Chitrakut and Mirzapur; area belonging to high category in between 5.38 and 8.38 per cent was seen in 4 districts of Balrampur, Siddharthnagar, Jhansi, and Sonbhandra having area in medium category in between 2.34 and 5.38 per cent in 7 districts of Moradabad, Bareilly, Badaun, Bahraich, Maharajganj, Hamirpur and Banda, Low area in between 0.75 and 2.24 per cent in 8 districts of Etah, Jalaun, Sitapur, Kushi Nagar, Basti, Gorakhpur, Allahabad and Chandauli.

Table 9: Other Sources Irrigated Area in Uttar Pradesh -2001-2005

\begin{tabular}{|c|c|c|c|}
\hline Category & Index range & $\begin{array}{c}\text { No. of } \\
\text { districts }\end{array}$ & Name of the district \\
\hline Very High & Above 8.38 & 4 & Mahoba, Lalitpur, Chitrakut and Mirzapur. \\
\hline High & $5.38-8.38$ & 4 & Balrampur, Siddharthnagar, Jhansi, and Sonbhandra \\
\hline Medium & $2.34-5.38$ & 7 & Moradabad, Bareilly, Badaun, Bahraich, Maharajganj, Hamirpur and Banda \\
\hline Low & $0.75-2.24$ & 8 & Etah, Jalaun, Sitapur, Kushi Nagar, Basti, Gorakhpur, Allahabad and Chandauli \\
\hline Very Low & Below 0.75 & 47 & $\begin{array}{c}\text { Saharanpur, Muzaffarnagar, Bijnor, Baghpat, Meerut, J. P. Nagar, Ghaziabad, G. B. Nagar, } \\
\text { Bulandshahar, Aligarh, Mathura, Hathras, Agra, Firozabad, Mainpuri, Farrukhabad, Etawah, } \\
\text { Kannauj, Aurauja, Rampur, Pilibhit Shahjahanpur, Kheri, Bahraich, Hardoi, Barabanki, } \\
\text { Lucknow, Unnao, Kanpur (rural), Kanpur (urban), Gonda, S. K. Nagar, Deoria, Faizabad, } \\
\text { Ambedkar Nagar, Azamgarh, Mau, Ballia, Sultanpur, Jaunpur, Ghazipur, Raebareli, Fatehpur, } \\
\text { Pratapgarh, Kaushambi, Sant Ravidas Nagar and Varanasi }\end{array}$ \\
\hline
\end{tabular}


Very low area under other source of irrigation below 0.75 per cent which cover more than half of the districts is found in 47 districts of Saharanpur, Muzaffarnagar, Bijnor, Baghpat, Meerut, J. P. Nagar, Ghaziabad, G. B. Nagar, Bulandshahar, Aligarh,

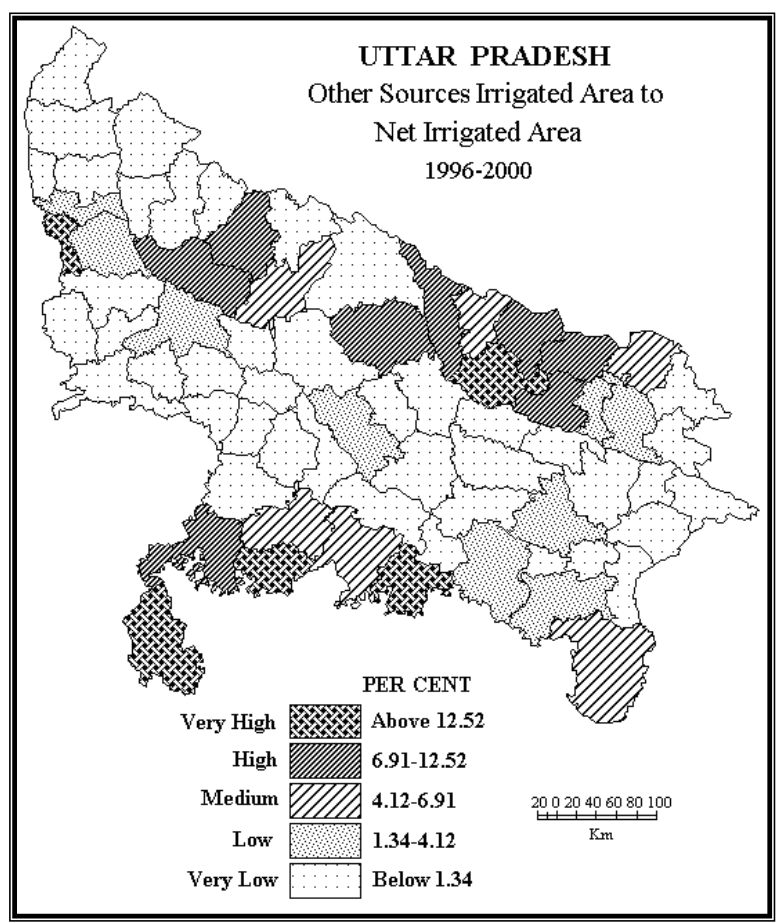

Source: Prepared by Author

Fig 8: Uttar Pradesh other sources irrigated area to net irrigated area 1996-2000

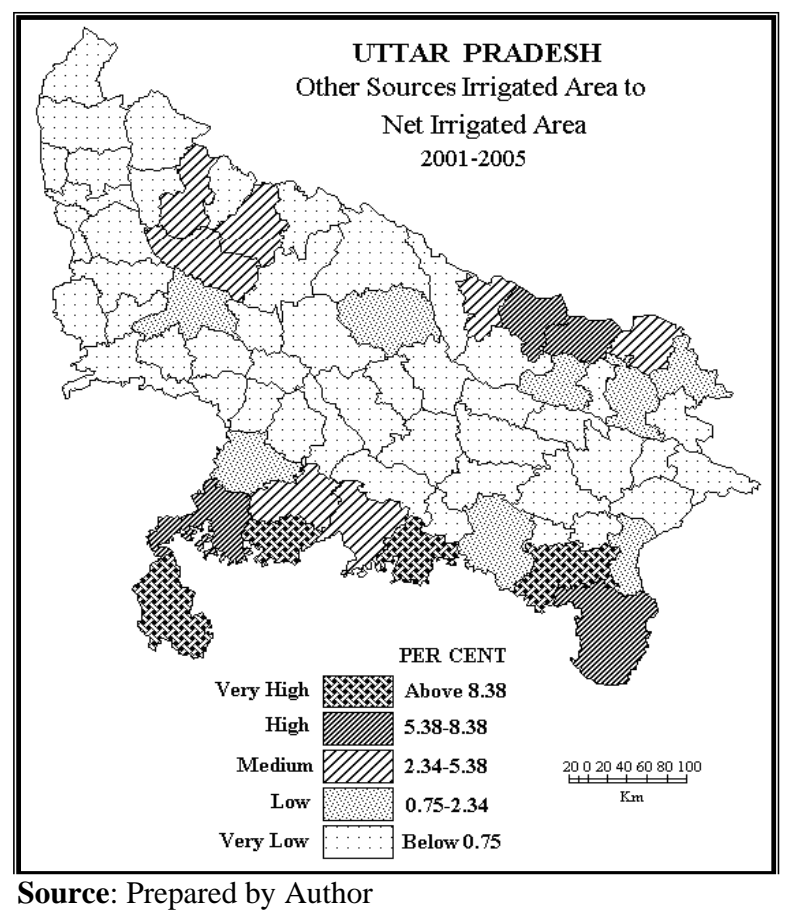

Fig 9: Uttar Pradesh other sources irrigated area to net irrigated area 2001-2005

Mathura, Hathras, Agra, Firozabad, Mainpuri, Farrukhabad, Etawah, Kannauj, Aurauja, Rampur, Pilibhit Shahjahanpur, Kheri, Bahraich, Hardoi, Barabanki, Lucknow, Unnao, Kanpur (rural), Kanpur (urban), Gonda, S. K. Nagar, Deoria, Faizabad, Ambedkar Nagar, Azamgarh, Mau, Ballia, Sultanpur, Jaunpur, Ghazipur, Raebareli, Fatehpur,
Pratapgarh, Kaushambi, Sant Ravidas Nagar and Varanasi which covered more than half of the districts of the state.

\section{Conclusion}

Present paper has discussed area under canal irrigation, area under tubwell irrigation and area under other source irrigation during 1996-2000 and 2001-2005 with respect to the net irrigated area. Western Uttar Pradesh has high availability of water for irrigation than Eastern Uttar Pradesh. Area under canal irrigation is marked high in the district of Jalaun, Banda, Chandauli, Mirzapur, Sonbhadra, Mathura, Etawah, Kanpur (rural), Kanpur (urban), Jhansi, Hamirpur, Mahoba, Barabanki, Raebareli, Pratapgarh, Allahabad, and Varanasi and Tube-well irrigation in Baghpat, J. P. Nagar, Rampur, Hathras, Kheri, Farrukhabad, Firozabad, Gonda, Basti, Gorakhpur Saharanpur, Meerut, Bulandshahar, Aligarh, Agra, Bareilly, Badaun, Shahjahanpur, Sitapur, Hardoi, Kannauj, Lucknow, Bahraich, Shrawasti, Balrampur, S. K. Nagar, Deoria, Faizabad, Ambedkar Nagar, Azamgarh, Mau, Ballia, Varanasi, S. R. Nagar and Kaushambi. It has been clear from above discussion that the districts of Bahraich, Siddharth Nagar, Gonda, Basti, G. B. Nagar, Jalaun, Jhansi Kanpur (urban), Fatehpur, Khushambi, Mirzapur Shrawasti, Balrampur, Hamirpur, Mahoba, Banda, Chitrakut and Sonbhadra needs attention to improve irrigation facilities.

\section{Suggestions}

- Government should take step to provide continuous electricity supply in rural areas for exploitation of groundwater irrigation in Eastern Uttar Pradesh.

- Groundwater recharge through rain water harvesting would help the farmers to grow their crops timely.

- Dry farming techniques in low rainfall and water scarcity areas can avert the ill effects of droughts.

- Area under irrigation should be expended can greatly increase agricultural productivity.

- The government should introduced some policies and programmes for the proper management and conservation of water resources in weaker districts of the state Uttar Pradesh.

\section{References}

1. Dhawan BD. Underutilisation of Groundwater Resources: A Case Study of East Uttar Pradesh, Vol. 15, No. 39, Economic and Political Weekly. 1980;A113-A115+A117-A122.

2. Govt of India. Irrigation and Power Projects, Ministry of Irrigation and Power, New Delhi 1970.

3. Govt. of India. Irrigation Atlas of India, New Delhi 1971.

4. Jain Bharatial H. Irrigation - Tapping natures bounty, The Hindu Survey of Indian Agriculture, Chennai 2004, 138-139.

5. Umar N. Impact of Green Revolution on Land Use and Crop Production in Uttar Pradesh, my thesis, Department of Geography, Aligarh Muslim University, Aligarh 2014.

6. NATMO. Irrigation Atlas of India, $2^{\text {nd }}$ Ed. National Atlas and Thematic Mapping Organization, Kolkata 1990.

7. Report of the Irrigation Commission. Vol. I, Ministry of Irrigation and Power, New Delhi 1972. 
8. Awasthi US. Fertilizer - Pragmatic views, concerns, The Hindu Survey of Indian Agriculture, Chennai, 2004, 145-146.

9. Khullar DR. A Comprehensive Geography, New Delhi. 296-302, 494-505,566-71.

10. Government of India. Report of the Expert Group on Groundwater Management and Ownership, Planning Commission, New Delhi, 2007.

11. Husain M. Systematic Agriculture Geography, New Delhi 2002, 360-364, 403-416.

12. Mishra Puri. Agricultural Inputs and Green Revolution, Indian Economy, 1995,490.

13. Pant Niranjan. "Trends in Groundwater Irrigation in Eastern and Western Uttar Pradesh”, Economic and Political Weekly, July, 2004, 3463-3468.

14. Reddy PS. Different sources of irrigation: a case study of the Telengana region, Manak Publications, Delhi, 1997.

15. Sivanappan RK. A proposed Action Programme to Maintain Groundwater Levels and Achieve Sustainable Agriculture in Tamil Nadu", News from the Fields, Groundwater development and lift irrigation, ODI Irrigation Management Network paper 5.Overseas Development Institute, London 1995.

16. Sharma P, Sharma RC. Factors Determining Farmers' Decision for Buying Irrigation Water: Study of Groundwater Markets in Rajasthan, Agricultural Economics Research Review, Vol. 19, January-June 2006, 39-56. 